\title{
Bronquiectasias en pacientes con secuela de tuberculosis
}

\author{
Bronchiectasis as sequelae in patients with pulmonar tuberculosis \\ Félix K. Llanos-Tejada ${ }^{1}$, Juan A. Salas-López ${ }^{2}$,Edwin H. Herrera-Flores ${ }^{3}$, \\ María Venero-Cáceres ${ }^{4}$, Carlos A. Saavedra-Leveau ${ }^{5}$
}

Resumen

Las bronquiectasias son un síndrome clínico, frecuente en nuestro medio, resultado de una serie de condiciones de inflamación persistente con daño estructural bronquial. Sus causas son múltiples, las que incluyen las secuelas de Tuberculosis Pulmonar. En el presente simposio, se considera su fisiopatología y clasificación con una explicación concisa de los mecanismos que la producen. En el apartado de diagnóstico, se enfoca sobre las características de las pruebas de imágenes que apoyan su definición; asimismo en las indicaciones de las pruebas de función pulmonar. Finalmente, en el apartado del tratamiento, se hará un resumen de las propuestas con evidencia científica que se tienen hasta al momento actual.

Palabras clave: Bronquiectasias, secuela, tuberculosis.

\section{Abstract}

Bronchiectasis is a clinical syndrome, common in our country, as a result of a series of conditions of persistent inflammation with bronchial structural damage. Its causes are multiple, which include the pulmonary tuberculosis sequelae. In this symposium, its pathophysiology and classification are covered, with a concise explanation of the mechanisms that produce it. In the diagnostic section, it is focused on the characteristics of the imaging tests that supports its definition; also the indications of pulmonary function tests are considered. Finally, a summary of the actual treatment proposals, under scientific evidences, were pointed out.

Keywords: Bronchiectasis, sequelae, tuberculosis.

\section{Introducción}

La primera descripción histopatológica de las bronquictasias fue realizada por Laënnec. Las bronquiectasias son dilataciones anormales, permanentes y generalmente irreversibles de los bronquios, y es el estado final de noxas persistentes en las vías aéreas de una serie de enfermedades sistémicas y/o locales del parénquima pulmonar. Pueden considerarse una neumopatía crónica de la vía aérea de mediano calibre ${ }^{(1-3)}$

Se caracterizan por un síndrome clínico de tos, esputo e infección bronquial, con una dilatación anormal y permanente de los bronquios, que se puede visualizar en estudios de imágenes.
Debido a que las bronquiectasias son el resultado de una serie de condiciones con inflamación persistente y daño estructural bronquial, sus causas pueden ser congénitas o adquiridas. Según estudios clínicos, el hallazgo de una causa secundaria varía de acuerdo al lugar geográfico, siendo más frecuentes las causas post infecciosas $(30 \%)$, las inmunodeficiencias $(5 \%)$, las asociadas a Enfermedad Pulmonar Obstructiva Crónica o EPOC (4\%), las asociadas a enfermedad del tejido conectivo (4\%), a disfunción mucociliar $(2 \%)$, y a aspergilosis alérgica broncopulmonar (2\%). En el $45 \%$ de los casos la causa no es reconocida, siendo catalogada como idiopática $^{(2-4)}$.

Se asume que esta proporción debe mantenerse en nuestro país, tomando especial importancia los casos

'Médico Neumólogo. Coordinador del Comité de Tuberculosis, Sociedad Peruana de Neumología. Instituto de Investigación en Ciencias Biomédicas. Facultad de Medicina. Universidad Ricardo Palma. Hospital Nacional Dos de Mayo. ${ }^{2}$ Médico Neumólogo del Hospital Nacional Dos de Mayo. profesor contratado de la Universidad Nacional Mayor de San Marcos. ${ }^{3}$ Médico Neumólogo y Especialista en Trastornos Respiratorios del Dormir. Coordinador del Comité de Trastornos Respiratorios del Dormir. Sociedad Peruana de Neumología. Hospital Nacional Arzobispo Loayza. ${ }^{4}$ Médico Neumólogo. Coordinadora del Comité de Fibrosis Pulmonar, Sociedad Peruana de Neumología. Hospital Nacional Arzobispo Loayza. ${ }^{5}$ Médico Neumólogo. Past Presidente Sociedad Peruana de Neumología. Director Académico. Facultad de Medicina, Universidad Nacional Mayor de San Marcos. Magister en Salud Ocupacional Yambiental. Doctor en Medicina. Facultad de Medicina San Fernando. Universidad Nacional Mayor de San Marcos.

Recibido: 18.11.2019 Aceptado: 09.12.2019 
secundarios a Tuberculosis Pulmonar y a Neumopatía Obstructiva secundaria a inhalación de humos de combustión orgánica (biomasa).

Los objetivos del tratamiento de las bronquiectasias son prevenir las exacerbaciones, disminuir los síntomas, mejorar la calidad de vida y detener la progresión de la enfermedad.

\section{Fisiopatología y Clasificación}

Los cambios estructurales que se han asociado, incluyen la dilatación bronquial, el engrosamiento de la pared bronquial y la impactación de moco. Asimismo, hay evidencia de existencia de enfermedad de la vía aérea pequeña y de enfisema, por lo que en más del $50 \%$ de los pacientes se llega a objetivar obstrucción del flujo aéreo, aunque también pueden observarse patrones restrictivos, mixtos y función pulmonar preservada $^{(4)}$.

El estímulo infeccioso es requisito primordial para su desarrollo. Al presentarse un patógeno, se genera la activación de células efectoras inmunes, predominantemente neutrófilos, que liberan proteasas, especies reactivas de oxígeno y otras citoquinas inflamatorias con el fin de combatir la infección. Estas a su vez lesionan el bronquio al producir inflamación transmural, edema asociado a ulceración mucosa, vascularización y en última instancia remodelación de la vía aérea con destrucción y dilatación anormal permanente de la pared del bronquio o bronquiolo ${ }^{(5-7)}$

La fisiopatología no está clara. Es posible que la infección viral inicial puede causar una disminución de la respuesta inmune local transitoria en algunos casos. En otros, puede ser por una prescripción inapropiada de corticoides inhalados o por bronquitis bacteriana persistente (PBB, por sus siglas en inglés) como primera presentación de una condición sistémica más severa. Es claro que el espectro de neumopatía supurativa crónica se extiende desde PBB hasta bronquiecta$\operatorname{sia}^{(4,8) \text {. }}$

Dados estos procesos, se propone un proceso fisiopato lógico plausible que refleja el ciclo vicioso original propuesto por Peter Coles ${ }^{(4,8)}$ : (i) Inflamación progresiva a predominio de neutrófilos que conduce a un daño adaptativo inadecuado de la pared como resultado de infección persistente o recurrente, con respuesta inmune mediada por Th17 que concluye en reclutamiento de neutrófilos e hipersecreción de moco; (ii) Estos factores comprometen aún más el aclaramiento mucociliar, promoviendo la oclusión de la vía aérea; y (iii) Resultante de la obstrucción se promueve la dilatación bronquial, con infección bacteriana persistente e inflamación crónica.

En definitiva, las variables clínicas solo pueden proporcionar poca información sobre la biología subyacente de la enfermedad. Recientemente, las investigaciones buscan comprender los genotipos, endotipos y el proceso de patobiología, mediante el uso de biomarcadores, lo que ofrece enfoques prometedores para desarrollar intervenciones que puedan permitir estratificar en términos de pronóstico y respuesta a la terapia ${ }^{(6,9)}$.

Existen distintos sistemas de clasificación de las bronquiectasias basados en patogénesis, etiología, factores predisponentes y hallazgos patológicos o anatómicos. La más reconocida y aceptada es la de Lynne Reid, que las agrupa según la morfología y severidad ${ }^{(10)}$.

1. Bronquiectasias tubulares o cilíndricas. Bronquios de contornos regulares, diámetro distal algo dilatado y la luz bronquial ocluida por tapones mucosos.

2. Bronquiectasias varicosas. Apariencia similar al de las venas varicosas. Presentan la vía aérea más dilatada con contornos irregulares por la presencia de constricciones fibrosas localizadas que le dan un aspecto irregular, tipo rosario.

3. Bronquiectasias saculares o quísticas. Presentan dilatación progresiva hacia la periferia de las vías aéreas y terminan en grandes quistes, sacos, o como racimos de uvas.

Esta clasificación correlaciona con la severidad clínica, aunque su asociación con la etiología de las bronquiectasias es pobre y poco orientadora.

\section{Diagnóstico}

Nuestros conocimientos sobre las causas se basan en el concepto del círculo vicioso descrito por Peter Coles, siendo los componentes claves de la enfermedad la infección bronquial crónica, la inflamación, la alteración de la limpieza mucociliar y el daño pulmonar estructural ${ }^{(1,3,9)}$.

Respecto a los estudios de imágenes, las bronquiectasias se presentan como bronquios dilatados, no afilados, de pared engrosada independientemente de los factores predisponentes y la etiología.

La radiografía de tórax es un estudio muy accesible y documenta hallazgos patológicos en más del $80 \%$ de los pacientes con bronquiectasias. Sin embargo, no es ni sensible ni específico. Los signos radiográficos sugestivos de bronquiectasias comprenden el "rail en tranvía", que son opacidades lineales paralelas correspondientes a los bronquios dilatados de pared engrosada; el "anillo de sello", que es una imagen del bronquio en que se observa bien definida su pared; y, por último, el "dedo de guante", que representa opacidades irregulares periféricas correspondientes a tapones mucosos bronquiales. La presencia de dichos signos por separado y aún más en conjunto, soportan el diagnóstico si se ven asociados a un cuadro clínica sugestivo ${ }^{(9,11)}$.

El método de imagen considerado en la actualidad como "estándar de oro" para el diagnóstico es la tomografía axial 
computarizada de alta resolución, en la cual los datos más específicos de bronquiectasias son la dilatación del bronquio objetivada como un diámetro bronquial mayor a 1.5 veces el diámetro de la arteria pulmonar adyacente, la falta de afilamiento bronquial que se manifiesta como la presencia de estructuras bronquiales a menos de $10 \mathrm{~mm}$ de la pleura parietal, y el patrón de árbol en gemación que indica afección de vía aérea pequeña ${ }^{(9,11)}$.

La distribución de las bronquiectasias en los estudios de imágenes pueden orientar hacia una etiología específica. Una distribución central es sugestiva de Aspergillosis broncopulmonar alérgica, Síndrome de Mounier Kuhn o Síndrome de Williams Campbell. Lesiones en lóbulos superiores son frecuentes cuando la enfermedad de fondo es Fibrosis quística o sus variantes. Lesiones en lóbulo medio e inferior orientan hacia Discinesia ciliar primaria. Lesiones en lóbulos medio y língula es característico de micobacterias no tuberculosas. Y únicamente en lóbulos inferiores sugieren bronquiectasias idiopáticas $^{(9,11,12)}$.

Se sugiere una cantidad mínima de exámenes etiológicos en los adultos con diagnóstico reciente de bronquiectasias. Estos son: 1) Recuento sanguíneo diferencial; 2) Inmunoglobulinas séricas (IgG, IgA e IgM totales); 3) Pruebas en búsqueda de la aspergilosos broncopulmonar alérgica (ABPA). Los cultivos de esputo se deben realizar con propósitos de monitorización de infección bacteriana. Otros exámenes adicionales podrían ser apropiados en respuesta a características clínicas específicas o en pacientes con enfermedad rápidamente progresiva.

Las pruebas de función pulmonar, particularmente el volumen espiratorio forzado al primer minuto (VEF1), son un marcador confiable de la reserva pulmonar y de respuesta al tratamiento. Por lo general se documenta un patrón obstructivo con o sin evidencia de hiperreactividad de la vía aérea. Los volúmenes pulmonares se encuentran normales, aumentados o disminuidos, y la capacidad de difusión de monóxido de carbono con frecuencia, está también disminuida, pero con gradiente alveolo-arteriolar aumentado ${ }^{(3,13,14)}$.

\section{Tratamiento}

El tratamiento se basa primariamente en los principios de prevención o supresión de la infección aguda y crónica, mejora de la limpieza mucociliar y disminución del impacto en la calidad de vida de la enfermedad estructural ${ }^{(3,9)}$.

La infección crónica estimula y mantiene la inflamación de la vía aérea. Esta infección es producida por Haemophilus influenzae y Pseudomonas aeruginosa y, menos frecuentemente, por Moraxella catarrhalis, Staphylococcus aureus y Enterobacteriaceae.

El aislamiento persistente de esos organismos se ha asociado a un incremento de las exacerbaciones, a una peor calidad de vida y a un aumento en la mortalidad; especialmente en la infección por Pseudomonas ${ }^{(1-3,15)}$.

La inflamación es primariamente neutrofílica y se asocia fuertemente con la infección bacteriana persistente.

Por otra parte, la limpieza mucociliar está alterada por el impacto estructural, por deshidratación de la vía aérea y por alteraciones del moco bronquial en volumen e incremento de la viscosidad. El tratamiento busca prevenir la ectasia del moco, la obstrucción al flujo aéreo mediante el uso de broncodilatadores y el daño del parénquima pulmonar progresivo; asimismo, mejorar la capacidad de ejercicio (con rehabilitación pulmonar) o remover el pulmón enfermo (cirugía).

El tratamiento se ha extrapolado del manejo de las bronquiectasias asociadas a la fibrosis quística, enfermedad poco frecuente en nuestro medio. Sin embargo, se sabe que las respuestas al tratamiento son diferentes y que son necesarias guías específicas para las bronquiectasias no fibrosis quística $(11,13,16)$

En ausencia de datos directos que comparen cursos más largos de antibióticos con cursos más cortos, se sugiere continuar la práctica usual de tratar las exacerbaciones con 14 días de antibióticos en base a la microbiología previa del paciente y la severidad de la exacerbación ${ }^{(2,3,9,12,13)}$.

Se sugiere que en casos de aislamiento de $P$. aeruginosa se debe ofrecer tratamiento antibiótico de erradicación. Se sugiere no ofrecer tratamiento antibiótico de erradicación a los adultos con aislamiento de patógenos que no sean la P. aeruginosa $\mathrm{a}^{(2,3,9,12,13)}$.

Y, aunque no se han establecido definiciones de infección crónica de la vía aérea, la definición más utilizada en los estudios es dos o más aislamientos del mismo organismo con al menos 3 meses de diferencia durante un año. Para el tratamiento de la inflamación bronquial, la evidencia respalda el tratamiento antibiótico a largo plazo, especialmente en casos con tres o más exacerbaciones al año, habiéndose determinado el beneficio con macrólidos (azitromicina, eritromicina) en caso de infección crónica por P. aeruginosa cuando el tratamiento con antibióticos inhalados esté contraindicado, no sea tolerado o no sea posible. Se puede ofrecer tratamiento a largo plazo con un antibiótico oral elegido de acuerdo a la susceptibilidad y tolerancia del paciente en caso los macrólidos están contraindicados, no sean tolerados o sean inefectivos $(2,3,9,12,13)$.

No existe evidencia que respalda el uso de corticoides inhalados, estos deben reservarse para casos con broncorrea persistente que no cede con el uso de antibióticos a largo plazo.

Puede brindarse tratamiento mucoactivo a largo plazo ( $\geq$ meses) en adultos con dificultad para expectorar con mala calidad de vida y no se hayan logrado un control de síntomas con las técnicas estándares de limpieza de la vía aérea mediante 
fisioterapia respiratoria. Se debe tener en cuenta que ninguno de los agentes mucoactivos estudiados disminuyó el número de exacerbaciones, tampoco hubo cambios en la función pulmonar y no hay evidencia suficiente que permita evaluar el uso de los mucolíticos orales. Bromhexina a dosis altas $(30 \mathrm{mg} / 8 \mathrm{~h}) \mathrm{se}$ podría utilizar en casos de expectoración diaria superior a 30 ml. En pacientes que además presentan EPOC, se podrían valorar otros mucolíticos, como acetilcisteína (dosis de 400$1800 \mathrm{mg} /$ día), carbocisteína (dosis de $15002700 \mathrm{mg} /$ día) o ambroxol (75 mg/día) a más largo plazo (> 10 meses), ya que podrían reducir las agudizaciones. Lo que sí es claro en la evidencia revisada es que la DNasa recombinante podría ser contraproducente ${ }^{(2,3,9,12,13)}$

Se debería reservar el uso de inhalación de broncodilatadores para aquellos casos con obstrucción del flujo aéreo demostrado por pruebas de función pulmonar y en casos de disnea sintomática que afecte la rutina diaria.

Los programas de rehabilitación pulmonar y el ejercicio regular se recomiendan en aquellos con capacidad de ejercicio alterada. Todas estas intervenciones deben ser individualizadas según los síntomas, capacidad física y características de la enfermedad ${ }^{(3,9)}$.

Existe un mayor riesgo de desnutrición, especialmente de masa magra, por presentar mayores requerimientos energéticos (por el aumento del trabajo respiratorio y la inflamación crónica) y por la anorexia, especialmente durante las agudizaciones. Al igual que en EPOC, el índice de masa corporal (IMC) $<20 \mathrm{~kg} / \mathrm{m} 2$ es un marcador independiente de supervivencia y la desnutrición de masa magra se asocia a complicaciones y mayor mortalidad. La valoración nutricional debe formar parte de la evaluación integral, siendo indispensable medir el IMC en cada visita y valorar la ingesta y la pérdida de peso en el tiempo ${ }^{(3,9)}$.

Finalmente, es importante también considerar y valorar el estado de salud mental y su impacto en la calidad de vida, por lo que el tratamiento integral debe incluir estas variables ${ }^{(15,17)}$.

\section{Conclusiones}

Las bronquiectasias son un conjunto de enfermedades cuya frecuencia no es conocida en nuestro medio, que posiblemente sea más común de lo que se describe por la presencia del antecedente de tuberculosis pulmonar.

El diagnóstico requiere evaluación de personal entrenado, estudio de imágenes y pruebas completas de función pulmonar.
Referencias bibliográficas

1.Martínez García MA. Bronquiectasias: ¿todavía una enfermedad huérfana? Arch Bronconeumol. 2005;41(8):407-409.

2.Acón Ramírez E, Rodríguez Sánchez O. TEMA 16-2015 Actualización en bronquiectasias. Rev Clínica Esc Med UCR-HSJD [Internet]. 6 de noviembre de 2015 [citado 14 de septiembre de 2019];5(5). Disponible en: http://revistas.ucr. ac.cr/index. $\mathrm{php} / \mathrm{clinica} /$ article/view/21710

3. Martínez-García MA, y col. Normativa sobre el tratamiento de las bronquiectasias en el adulto. Arch Bronconeumol. 2018:54(2);88-98.

4.Hill AT, Chang AB. Moving forward: Bronchiectasis and chronic suppurative lung disease in children and adults in the 21 st century: Series Editorial-Prologue. Respirology. 2018;23(11):1004-1005.

5. Bush A, Floto RA. Pathophysiology, causes and genetics of paediatric and adult bronchiectasis: Mechanisms and causes of bronchiectasis. Respirology [Internet]. 25 de febrero de 2019 [citado 14 de septiembre de 2019]; Disponible en: http://doi.wiley.com/10.1111/resp.13509

6. King PT. The Role of the Immune Response in the Pathogene- sis of Bronchiectasis. BioMed Res Int. 2018;2018:1-12.

7. Green H, Jones A. The Microbiome and Emerging Pathogens in Cystic Fibrosis and Non-Cystic Fibrosis Bronchiectasis. Semin Respir Crit Care Med. 2015;36(2):225-235

8. Mitchell AB, Mourad B, Buddle L, Peters MJ, Oliver BGG, Morgan LC. Viruses in bronchiectasis: a pilot study to explore the presence of community acquired respiratory viruses in stable patients and during acute exacerbations. BMC Pulm Med. 2018;18(1):84.

9. Chotirmall SH, McShane PJ. Time to acknowledge, address, and take action against bronchiectasis. Lancet Glob Health. 2019;7(9):e1162-3.

10. Reid LMcA. Reduction in Bronchial Subdivision in Bronchiectasis. Thorax. 1950;5(3):233-247.
11. Schäfer J, Griese M, Chandrasekaran R, Chotirmall SH, Hartl D. Pathogenesis, imaging and clinical characteristics of $\mathrm{CF}$ and non-CF bronchiectasis. BMC Pulm Med. 2018;18(1): 79 .

12. Lesan A, Lamle AE. Short Review on the Diagnosis and Treatment of Bronchiectasis. Med Pharm Rep [Internet]. 21 de diciembre de 2018 [citado 14 de septiembre de 2019]; Disponible en: h t t p s : // w w w. medpharmareports. com/index. $\mathrm{php} / \mathrm{mpr} / \mathrm{article} / \mathrm{view} / 1060$

13. Chalmers JD, Crichton M, Goeminne PC, Loebinger MR, Haworth C, Almagro M, et al. The European Multicentre Bronchiectasis Audit and Research Collaboration (EMBARC): experiences from a successful ERS Clinical Research Collaboration. Breathe. 2017;13(3):180-192.

14.Flume PA, Chalmers JD, Olivier KN. Advances in bronchiectasis: endotyping, genetics, microbiome, and disease heterogeneity. The Lancet. 2018:392(10150):880-890.

15. O'Leary CJ, Wilson CB, Hansell DM, Cole PJ, Wilson R, Jones PW. Relationship between psychological well-being and lung health status in patients with bronchiectasis. Respir Med. 2002;96(9):686-692.

16.Dhar R, Singh S, Talwar D, Mohan M, Tripathi SK, Swarnakar R, et al. Bronchiectasis in India: results from the European Multicentre Bronchiectasis Audit and Research Collaboration (EMBARC) and Respiratory Research Network of India Registry. Lancet Glob Health. 2019;7(9):e1269-1279.

17. Girón Moreno RM, Fernandes Vasconcelos G, Cisneros C, GómezPunter RM, Segrelles Calvo G, Ancochea J. Trastornos psicológicos y calidad de vida en pacientes con bronquiectasias no relacionadas con fibrosis quística. Arch Bronconeumol. 2013;49(10):415-420.

Citar como: Llanos-Tejada FK. y cols. Bronquiectasias en pacientes con secuela de tuberculosis. Diagnóstico(Lima).2019;58(4):190-193

DOI: https://doi.org/10.33734/diagnostico.v58i4.175

Correspondencia: Félix K. Llanos-Tejada. Correo electrónico: felex3d@yahoo.com 